\title{
Mammary Gland Studies as Important Contributors to the Cause of Epithelial Mesenchymal Plasticity in Malignancy
}

\author{
H. L. Ford • E. W. Thompson
}

Published online: 11 June 2010

(C) Springer Science+Business Media, LLC 2010

Over the last few years, the field of epithelial-mesenchymal transition (EMT) has rapidly expanded, both in developmental biology and in disease pathology (Fig. 1a). The growing literature suggests that this field may perhaps more appropriately be referred to as epithelial-mesenchymal plasticity (EMP), in light of the increased evidence for the reverse transition (MET), and for the 'hybrid state', in which cells express both epithelial and mesenchymal markers. Breast cancer research has always been well represented in the cancer EMT literature (Fig. 1b) and studies of EMT in normal mammary function that may relate to tumour biology are emerging and are adding to the total number of mammaryrelated EMT papers (Fig. 1c). This Special Issue entitled "EMT in Mammary Development and Breast Cancer Progression" captures much of the current themes in mammary biology and EMP, arranged somewhat chronologically.

It begins with a description of the EMT programs utilized in embryologic development (Ford and colleagues), then moves on to polarity mechanisms and the ways in which these are regulated during developmental EMT

\footnotetext{
H. L. Ford $(\bowtie)$

Department of Obstetrics and Gynecology,

University of Colorado School of Medicine,

Aurora, CO, USA

e-mail: Heide.Ford@ucdenver.edu

E. W. Thompson

St. Vincent's Institute,

Fitzroy, VIC 3065, Australia

E. W. Thompson

Department of Surgery, St. Vincent's Hospital,

University of Melbourne,

Fitzroy, VIC 3065, Australia
}

(Humbert and colleagues), followed by a detailed description of EMT regulation by the SNAIL family of developmental transcription factors (Garcia-de Hererros and Savagner). It then continues with a historical perspective of EMT and a description of some of the mouse mammary models that exhibit EMT (Cardiff). Following this perspective, the special issue moves into the regulation of EMT by growth factors including the TGF- $\beta$ (Schiemann and colleagues) and EGF (Strizzi and colleagues) families, as well as the regulation of EMT by other critical players, including MMPs (Radisky) and the fascinating and more recently described microRNAs (miRs; Goodall and colleagues). In the final set of articles within this special issue, the relationship between EMP and the breast cancer stem cell (BCSC) phenotype (Thompson and colleagues) is discussed, including the clinical implications of this connection that have been so revealing of late (Rosen and colleagues). This leads directly into the last article of the issue, in which the prospect of EMT in the circulating tumour cells (CTC) that predispose patients to metastasis is discussed (Gilles and colleagues). It is heartening that several authors took up the suggestion to invite other leaders with similar interests to join their article, as seen with Garcia-de Hererros and Savagner on Snail, Goodall and Richer on miRs, Thompson, Mani and Weinberg on BCSC and Gilles and Thompson on relationships between EMP and CTC. This is not always easy, and we thank these authors for their willingness to participate in this manner and to effectively broaden the scope of this collection further than could have otherwise been done due to issue size limitations. Certainly such arrangements result in a more comprehensive and balanced review of the current literature.

In addition to their specific focus, many of the authors provide their slightly different description of current issues 
Figure 1 a Number of EMT-related articles published over the period from 2000-2009. b Number of breast cancer-related EMT and cancerrelated EMT articles published from 2000-2009. c Number of total mammary-related EMT (both normal mammary and cancer) to total EMT articles published from 2000-2009. Numbers determined by searching the Pubmed database on May 1, 2010. Search terms: epithelial, mesenchymal, transition, $+/-$ cancer. $+/-$ mammary.

in EMP biology that are particularly relevant to the mammary gland, which is insightful and refreshing to the extent that it overpowers any redundancy. Cooperativity, diversity and integrational cross-talk are commonly reported and described, as are contextual constraints to the extent that Snaill can either repress or stimulate EMT depending on the cellular context. We envision that this theme may be more generally applicable, as context can certainly dictate phenotype. The roles and manifestations of EMT in mammary tumorigenesis are touched on by almost all authors, and the reverse transition (MET) is discussed by quite a few. Blick et al. use the term epithelial-mesenchymal plasticity (EMP) to define a spectrum of change along the EpithelialMesenchymal axis, concordant with the partial EMT/metastable state described by Ford and colleagues (Micalizzi et al.), revealed in mouse models by Cardiff, and described in human tissues by Savagner and Garcia de Hererros: Lobular carcinoma being a particular case in point for this phenomenon (Savagner). Gilles, Thompson and co-workers (Bonnenet et al.) postulate that EMT-like changes will enable the generation, survival and progression of circulating tumour cells (CTCs), which could present a problem for current technologies that utilize epithelial markers to identify such cells in the circulation if the hybrid phenotype is not dominant. Importantly, Creighton et al. have shown the continued expression of cytokeratin in cells adopting mesenchymal features after neo-adjuvant systemic therapy.

Indeed, the clinical implications of EMP in breast cancer biology are profound, encompassing not only the problem of identifying CTCs that was outlined above. Blick et al. highlight that the basal $\mathrm{B}$ cell lines currently used in the laboratory are useful tools for developing molecular markers that can be utilized for detection and quantification of EMT and cancer stem cell phenotypes, which are associated with more aggressive cancers. Identification of cancers that have more EMT and stem cell features may allow for different and/ or more aggressive treatment strategies. In support of the argument that new treatment strategies may be needed in breast cancer, Creighton, Chang, and Rosen review the findings that certain commonly used breast cancer treatment strategies enrich tumors for cancer stem-like or tumor initiating cells, and that these cells have characteristics of EMT. Thus, targeting proteins that are important in EMT may be a useful strategy for preventing chemoresistance and

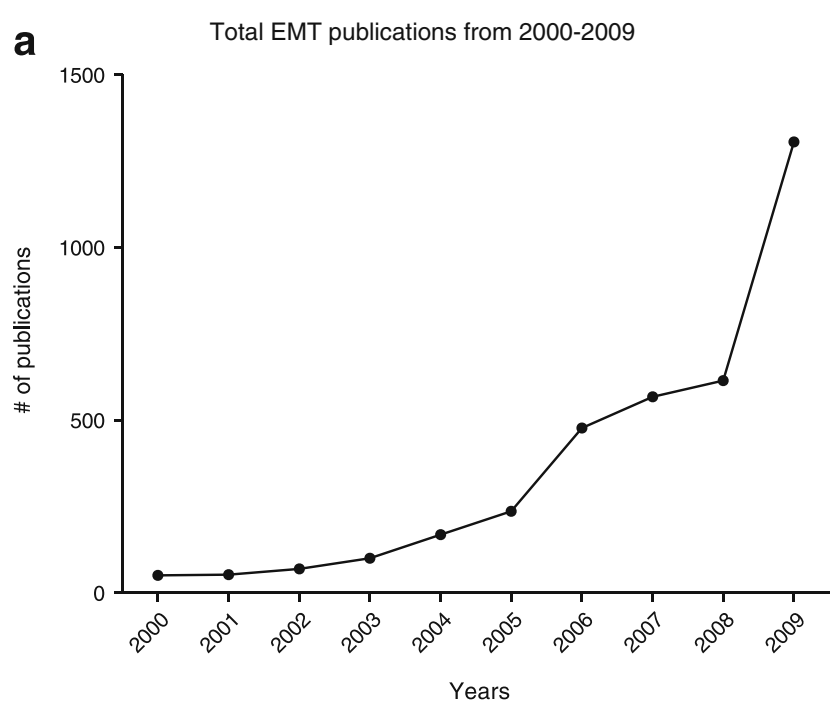

b Number of breast cancer EMT to total cancer EMT publications
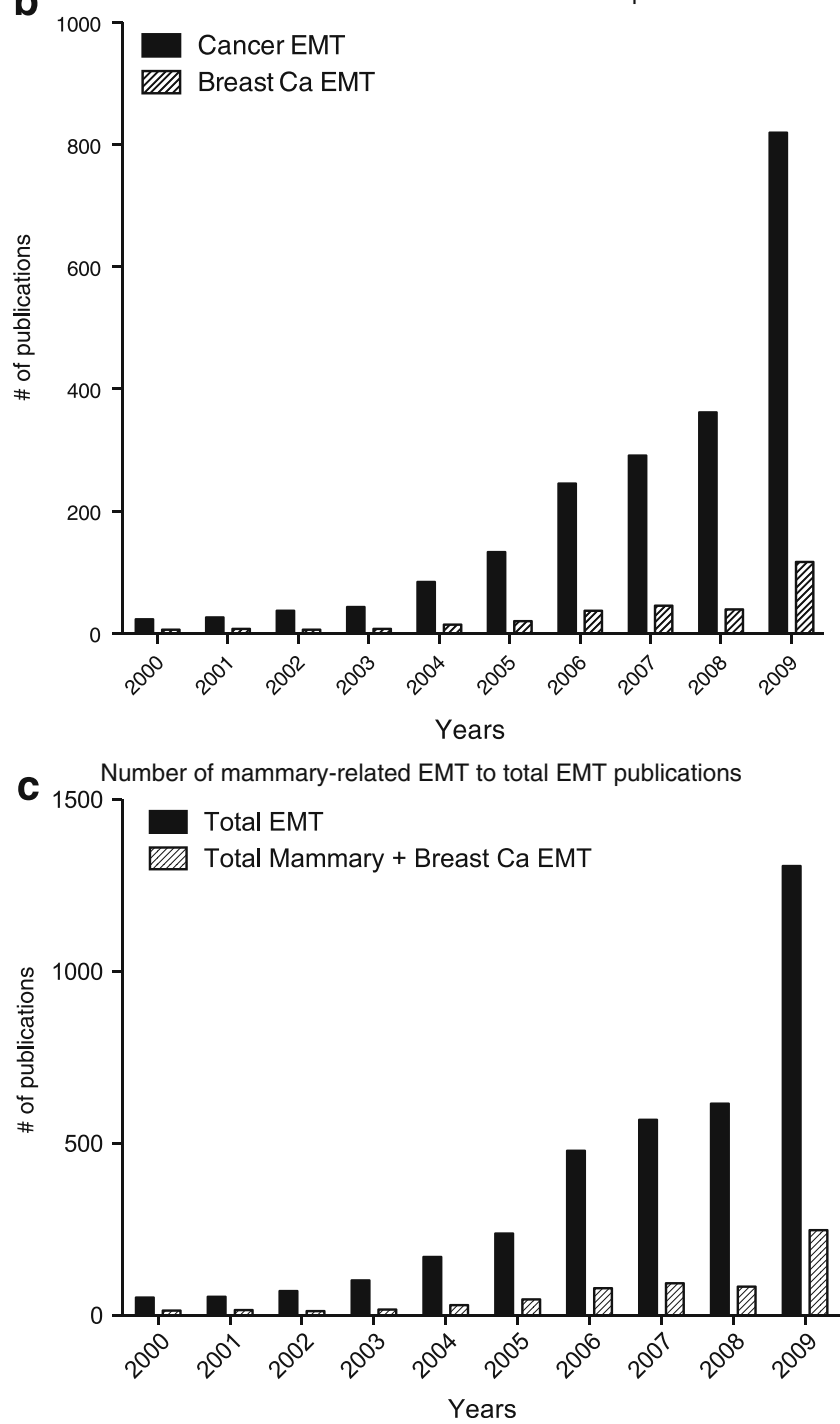
recurrence. These final clinical papers underscore how critical it is for the field to continue moving forward at an even more rapid pace than it has before.

Collectively, the field of EMP offers a new spectrum of opportunity in breast cancer treatment. The concrete revelations of EMP in clinical specimens, initially by Weinberg and coworkers [1], but more recently by Chang and co-workers [2] have answered the requirement for formal proof [3-5], and brought credence to the many reports implicating EMP-associated factors in breast cancer. The articles herein describe in great detail the myriad of approaches towards a better understanding of EMP and the identification of target molecules and pathways that may find utility in the diagnosis and treatment of breast cancer.

\section{References}

1. Mani SA et al. The epithelial-mesenchymal transition generates cells with properties of stem cells. Cell. 2008;133:704-15.

2. Creighton CJ et al. Residual breast cancers after conventional therapy display mesenchymal as well as tumor-initiating features. Proc Natl Acad Sci USA. 2009;106:13820-5. doi:10.1073/pnas. 0905718106

3. Christiansen JJ, Rajasekaran AK. Reassessing epithelial to mesenchymal transition as a prerequisite for carcinoma invasion and metastasis. Cancer Res. 2006;66:8319-26. doi:10.1158/0008-5472. CAN-06-0410.

4. Tarin D, Thompson EW, Newgreen DF. The fallacy of epithelial mesenchymal transition in neoplasia. Cancer Res. 2005;65:59966000. discussion 6000-5991.

5. Thompson EW, Newgreen DF, Tarin D. Carcinoma invasion and metastasis: a role for epithelial-mesenchymal transition? Cancer Res. 2005;65:5991-5. discussion 5995. 